\title{
Rotating flow of a nanofluid due to an exponentially stretching surface with suction
}

\begin{abstract}
An analysis of the rotating nanofluid flow past an exponentially stretched surface with the presence of suction is studied in this work. Three different types of nanoparticles, namely, copper, titania and alumina are considered. The system of ordinary differential equations is computed numerically using a shooting method in Maple software after being transformed from the partial differential equations. This transformation has considered the similarity transformations in exponential form. The physical effect of the rotation, suction and nanoparticle volume fraction parameters on the rotating flow and heat transfer phenomena is investigated and has been described in detail through graphs. The dual solutions are found to appear when the governing parameters reach a certain range.
\end{abstract}

Keyword: Rotating nanofluid flow; Stretching surface; Suction 